\title{
Design and characteristics of widely tunable quantum-well laser diodes
}

\author{
V.K. Kononenko ${ }^{a, 1}$, I.S. Manak ${ }^{b}$, S.V. Nalivko ${ }^{b}$ \\ ${ }^{a}$ Stepanov Institute of Physics, National Academy of Sciences of Belarus, \\ Fr. Scorina Pr., 70, 220072 Minsk, Belarus \\ ${ }^{b}$ Belarussian State University, Fr. Scorina Pr., 4, 220050 Minsk, Belarus
}

Received 9 November 1998, accepted 12 March 1999

\begin{abstract}
Light-current and tuning characteristics of semiconductor injection lasers in an external cavity are analyzed. The active region of the lasers is an asymmetric multiple quantum-well heterostructure under conditions of non-uniform excitation. Such a design of laser diodes results in a wide range tunable emission spectrum.
\end{abstract}

Keywords: External cavity; Laser diode; Quantum-well heterostructure; Tuning curve

\section{Introduction}

Effective continuously tunable laser diodes with a narrow spectral line cover a wavelength range from 630 up to $1570 \mathrm{~nm}$ [1]. Laser diodes are successfully applied in spectroscopic analysis and environmental monitoring instead of hollow-cathode lamps and more expensive and complex dye and solid-state laser sources [2-5]. Tunable laser diode sources distinguish by compactness, reliability, absence of complex optics, low price, easy maintenance, long lifetime, fairly monochromatic beam, and high stability of the output signal. The use of diffraction gratings as dispersing elements in external selective cavities gives opportunity to enhance the spectral brightness and stability of tunable laser diode sources. High qualities of laser diodes guarantee required spectral resolution and sensitivity at the realization of spectroscopy measurements.

Absorption lines of most gases are located in the emission spectral range of injection lasers. For more accurate coincidence of the absorption lines and output radiation, it is possible to control emission wavelengths of heterostructure lasers selecting an active region material, the Q-factor of the cavity, the pump current and temperature [6]. In quantum-well lasers there are additional opportunities of the lasing wavelength control by the change of thicknesses and component compositions of active and barrier layers [7,8]. The tuning range is determined by the width of the gain spectrum and hence it is rather narrow in these cases. Widening the gain spectrum is possible due to a novel conception of asymmetric quantum-well heterostructures where the potential profile in barrier regions and arrangement of differ active layers relative to current emitters play an essential role $[9,10]$.

In the present paper, analysis of lasing processes, light-current and tuning characteristics of an asymmetric multiple quantum-well heterostructure laser in an external cavity is performed. At first, design of the laser diode structure is discussed. Then, a theoretical model for description of lasing processes is considered. In the next section, configuration and operation principles of the external cavity are

\footnotetext{
${ }^{1}$ Corresponding author, e-mail: lavik@dragon.bas-net.by
} 
described. Finally, numerical calculations of light-current characteristics and tuning curves are presented.

\section{Laser diode structure}

The band diagram of the proposed asymmetric multiple quantum-well heterostructure laser under forward bias is shown in Fig. 1. The heterostructure component composition is connected with the GaAs-AlGaAs system. The active region of the laser consists of four quantum wells with widths $d_{1}=150 \AA, d_{2}=45 \AA$, $d_{3}=50 \AA$, and $d_{4}=90 \AA$. Different quantum wells give certain contributions to the total gain in various intervals of the spectrum. To make equal the gain contributions of the quantum wells, conditions of non-uniform excitation in the structure are fulfillment due to definite doping with donors and acceptors in barrier regions. At the forward bias the potential barrier appeared in the conduction band due to p-doping of the barrier layer between quantum wells 1 and 2 partly blocks the electron transport into quantum well 1 . The potential barrier arose in the valence band due to n-doping of the barrier layer between quantum wells 3 and 4 partly prevents from the hole transport into quantum well 4. As a result, non-uniform excitation of the quantum wells is achieved. In this case, the excitation level of narrow quantum wells 2 and 3 is enough high as compared with the excitation of wide quantum wells 1 and 4 .

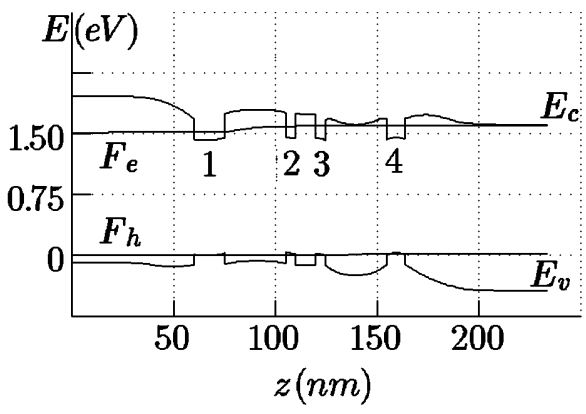

Fig. 1. Band diagram $E(z)$ of an asymmetric multiple quantum-well heterostructure laser at the forward bias of $1.6 \mathrm{~V} . F_{e}$ and $F_{h}$ are the quasi-Fermi levels for electrons and holes, $E_{c}$ and $E_{v}$ are the energies of the conduction band bottom and the valence band top, respectively.
In such a laser structure it is possible to control the efficiency of current injection into quantum wells at changing the barrier layer parameters (thickness, component compositions, level and type of doping). Parameters of active layers are chosen so that the wavelengths of the radiative recombination of electrons and heavy and light holes in different quantum wells were some shifted and covered a desirable range of the spectrum. It provides conditions for the achievement of continuous wavelength tuning in a rather broad interval.

\section{Theoretical background}

Analysis of radiative processes in the multiple quantum-well heterostructure laser has been provided using the single-mode rate equations

$\frac{d n_{i}}{d t}=\frac{\eta_{i} j}{e}-R_{s p i}-\frac{v g_{i} S}{1+\alpha_{i} \Gamma_{i} S}$,

$\frac{d S}{d t}=v\left(\sum_{i} \frac{g_{i}}{1+\alpha_{i} \Gamma_{i} S}-k_{l}\right) S+\beta \sum_{i} R_{s p i}$.

Here, $t$ is the time, $n_{i}$ is the twodimensional electron concentration in $i$-th quantum well, $S$ is the photon density for a lasing mode, $j$ is the pump current density, $\eta_{i}$ is the injection efficiency at the excitation of $i$-th quantum well, $R_{s p i}$ is the spontaneous recombination rate, $\beta$ is the factor determining a contribution of the spontaneous radiation to the lasing mode, $v$ is the light velocity in the crystal, $g_{i}$ is the modal gain in $i$-th quantum well at the lasing wavelength, $\alpha_{i}$ is the suppression factor connected with an additional reduction of the modal gain at the lasing wavelength with increasing the photon density in the cavity, $\Gamma_{i}$ is the optical confinement factor, $k_{l}$ is the losses of radiation.

For the sake of simplicity, values of the suppression factors $\alpha_{i}$ in different quantum wells are considered positive and to be of the same value [11]. The modal gain is calculated in a model of direct optical transitions in view of the emission line 


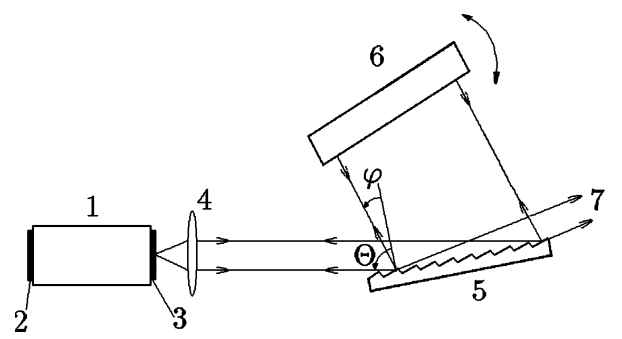

Fig. 2. Schematic diagram of the Littman and Metcalf external cavity configuration, (1) laser diode, (2) highly-reflective coating, (3) antireflection coating, (4) collimating lens, (5) diffraction grating, (6) tuning mirror, (7) output beam.

broadening because of finiteness of the intraband relaxation time of nonequilibrium current carriers [12]. Polarization peculiarities of optical transitions involving heavy and light holes are included too [10]. Following calculations are performed for the TE mode. In accordance with [13], the narrowing of the semiconductor band gap versus the level of excitation can be taken into account as well. The injection efficiencies $\eta_{i}$ have been calculated from the self-consistent solution of Poisson's equation and continuity equations for electron and hole currents.

\section{Spectral control by an external cavity}

There are several schemes of laser external cavities for the wavelength tuning [14-16]. As an example of selective external cavities, the modified Littman and Metcalf configuration [1] will be considered (Fig. ?). In this case, the front facet of a semiconductor laser crystal on the diffraction grating side has an anti-reflection coating and the other facet of the crystal is passivated with a highly-reflective coating. In the configuration, the diffraction grating is used at grazing incidence conditions and the optical feedback proceeds by a tuning mirror at the first-order diffraction. The output of the radiation occurs at the zeroorder diffraction angle. The lasing wavelength is controlled by rotation of the mirror.
The basic equation for the external cavity with a diffraction grating is [14]

$m \lambda=b(\sin \Theta+\sin \varphi)$,

where $\lambda$ is the wavelength of the output radiation, $b$ is the groove spacing, $m$ is the diffraction order, $\Theta$ is the angle between the grating normal and incident beam, $\varphi$ is the angle between the grating normal and diffracted beam. The spectral bandwidth of this laser spectrometer system $\Delta \lambda$ is determined according [14] in the doublepass approach by the grating input angle $\Delta \Theta_{i n}$ and the effective exit angle $\Delta \Theta_{e x}$, i. e.,

$$
\Delta \lambda=\frac{\lambda \cos \Theta}{2(\sin \Theta+\sin \varphi)} \sqrt{\left(\Delta \Theta_{i n}\right)^{2}+\left(\Delta \Theta_{e x}\right)^{2}} .
$$

Practically, the angular spread of the collimated intracavity beam having a Gaussian intensity profile is related to the diffraction divergence [1]. Therefore, the input half angle equals $\Delta \Theta_{i n} \approx \lambda / \pi R$, where $R$ is the radius of the beam. Since the width of the active waveguide region of the laser diode $d$ is small as compared with $R$, the exit half angle is equal to $\Delta \Theta_{\mathrm{ex}}=d / 2 F$, where $F$ is the lens focal length. This value is much smaller than $R / L$ which determines the possible deviation of the beam at the pass of the distance $L$ between the grating and the lens. Taking into account these facts we obtain

$\Delta \lambda=\frac{\lambda b}{\pi W} \sqrt{1+\left(\frac{\pi R d}{2 \lambda F}\right)^{2}}$,

where $W$ is the width of the illuminated part of the grating. As a rule, because of $\pi R^{2} / \lambda>L$, diffraction at the cavity length $L$ is relatively insignificant, i. e., $W \approx 2 R / \cos \Theta$.

As seen from Eq. (5), the spectral band width of the laser system is mainly determined by the input characteristics of the grating. For typical grating parameters and conditions of the laser spectrometer operation, e. g., $b=556 \mathrm{~nm}, \Theta=85^{\circ}$, $\varphi=30^{\circ}$, and $m=1$, one obtains $\lambda \approx \mathrm{nm}$. 
The laser waveguide region includes, besides the quantum well, barrier and cladding layers and its width approximately equals $d \approx 250 \mathrm{~nm}$. If $W=3 \mathrm{~cm}$, the collimated beam has the radius of about $1.3 \mathrm{~mm}$. One can put $F=3 \mathrm{~mm}$ and $L=5 \mathrm{~cm}$. In this case, we obtain $\Delta \lambda \approx 0.005 \mathrm{~nm}$. As seen, the output of radiation comes in a narrow spectral passband and the laser oscillates in a single mode in the whole tuning range. Obviously, multipass effects and the introduction of additional dispersion elements into the cavity reduce the spectral bandwidth of the laser spectrometer further.

At the analysis of the laser tuning characteristics, the intensity distribution versus the diffraction angle $I(\varphi)$ has been calculated according $[17,18]$. In normalized form taking into account the "shadow" geometrical effect in the grating we find

$$
I=\frac{b^{2}}{W^{2}} A^{2} \frac{\sin ^{2} u}{u^{2}} \frac{\sin ^{2}\left(\frac{W}{b} v\right)}{\sin ^{2} v},
$$

where $\delta$ is the blaze angle,

$$
\begin{aligned}
& u=\frac{\pi b}{\lambda} B(\sin (\Theta-\delta)+\sin (\varphi-\delta)), \\
& v=\frac{\pi b}{\lambda}(\sin \Theta+\sin \varphi),
\end{aligned}
$$$$
A=\frac{\cos \Theta \cos (\varphi-\delta)}{\cos (\Theta-\delta)}, \quad \Theta \geq \delta, \Theta \geq \varphi \geq-\pi 2+\delta,
$$

$\begin{array}{cc}A=\cos \varphi, & \varphi \geq \Theta \geq \delta,-\pi t 2+\delta \leq \Theta \leq \delta \leq \varphi(9) \\ A=\cos \delta \cos (\varphi-\delta), & \Theta \leq \delta, \varphi \leq \delta,\end{array}$

$B=\frac{A}{\cos (\varphi-\delta)}$.

The coefficient of the optical losses in the cavity is determined as

$$
k_{l}=\rho+\frac{1}{2 l} \ln \frac{1}{r_{1} r_{e f f}},
$$

where $\rho$ is the internal optical losses, $l$ is the laser diode length, $r_{1}$ is the reflectivity of the highly-reflective front facet of the semiconductor crystal, $r_{e f f}$ is the effective reflectivity of the equivalent cavity system consisted from the anti-reflection coated facet of the laser diode, the grating, and the tuning mirror (Fig. 2). The quantity $r_{\text {eff }}$ can be evaluated in the three-mirror cavity theory as

$r_{e f f}=r_{2}+\left(1-r_{2}\right)^{2} \frac{r_{3}}{1-r_{2} r_{3}}$.

Here, $r_{2}$ is the reflectivity of the crystal facet with the anti-reflection coating. The quantity $r_{3}$ determines the radiation portion came back onto the laser diode after diffraction at the grating, the reflection from the tuning mirror, and second diffraction at the grating. Hence, it equals $r_{3}=r_{g r 1} r_{m} r_{g r 2}$, where $r_{m}$ is the reflectivity of the tuning mirror,
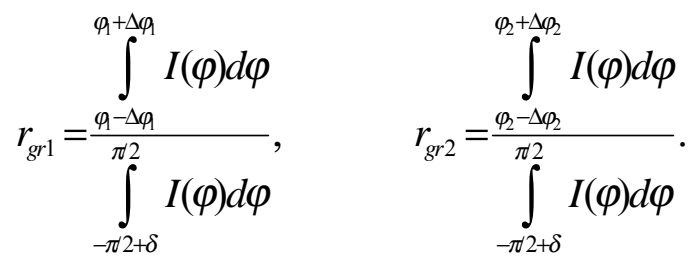

Here, $\varphi_{1}$ is the first-order diffraction angle at the first step of diffraction, $\Delta \varphi_{1}$ is the angle between the directions on the firstorder diffraction maximum and on the neighboring minimum, $\varphi_{2}$ is the first-order diffraction angle at the second step of diffraction, $\Delta \varphi_{2} \approx \Delta \Theta_{e x}=d / 2 F$. At the calculations of $r_{g r 2}$ the incident angle is consider to be equal to $\varphi_{1}$. The output coefficient $r_{0}$ determining the portion of the radiation pass to the output beam can be calculated by the formula (13) for $r_{g r 1}$ at the replacement of $\varphi_{1}$ on $-\Theta$ and of $\Delta \varphi_{1}$ on $\Delta \varphi_{0}$, where $\Delta \varphi_{0}$ is the angle between the directions on the zero-order diffraction maximum and on the neighboring minimum. Below, we assume that $r_{1} \approx 1, r_{2} \approx 0$, and $r_{m} \approx 1$. The dependencies of $r_{3}$ and $r_{0}$ on the lasing wavelength $\lambda$ which varies with $\varphi$ are shown in Fig. 3.

As seen, the efficiency of the optical feedback at the lasing wavelength in the 


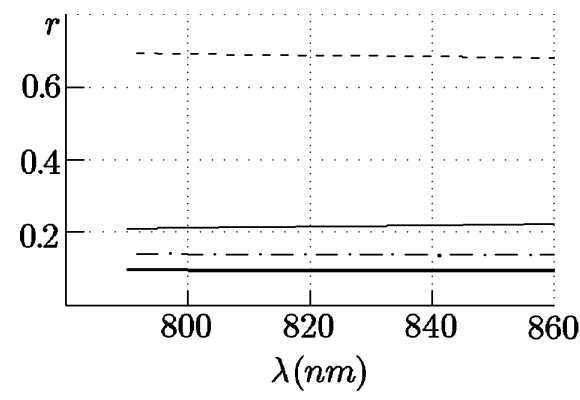

Fig. 3. Dependencies of the grating reflectivities $r=r_{\mathrm{gr} 1}(--), r_{\mathrm{gr} 2}(-.-), r_{3}(-)$, and $r_{0}(-)$ on the lasing wavelength $\lambda$.

modified cavity with the grating does not change greatly. The variation of the losses $k_{1}$ at tuning the lasing wavelength from 790 to $860 \mathrm{~nm}$ is only $0.5 \mathrm{~cm}^{-1}$. The following parameters of the system $b=556 \mathrm{~nm}$, $W=15 \mathrm{~mm}, \quad \delta=8^{\circ}, \quad \Theta=70^{\circ}, \quad \rho=5 \mathrm{~cm}^{-1}$, $l=250 \mu \mathrm{m}, d=250 \mathrm{~nm}, \quad F=2.5 \mathrm{~mm}$, and $L=5 \mathrm{~cm}$ were used.

\section{Light-current and tuning characteristics}

The output power of the laser system $P$ is related to the sheet photon density $S$ by the formula

$$
P=h v v l w S \frac{r_{0} k_{r}}{1+\frac{1-r_{1}}{1-r_{\text {eff }}}\left(\frac{r_{\text {eff }}}{r_{1}}\right)^{1 / 2}},
$$

where $v=c / \lambda$ is the light frequency, $k_{r}=(1 / 2 l) \ln \left(1 / r_{1} r_{\mathrm{eff}}\right), w$ is the width of the diode stripe contact. Given relation

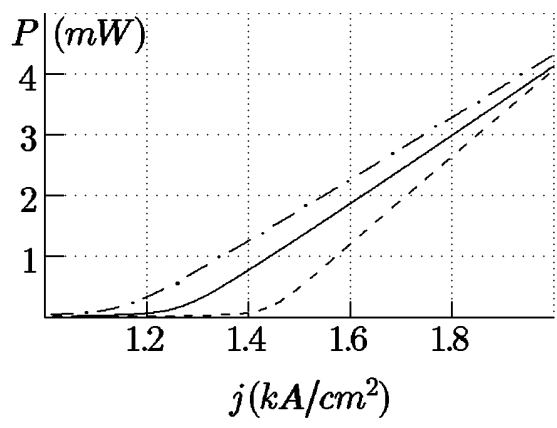

(a) transforms to the standard formula for the output power of laser diodes when $r_{\text {eff }}=r_{2}$ and $r_{0}=1$. The light-current characteristics calculated from the stationary rate Eqs. (1) and (2) with the account of Eq. (14) are shown in Fig. 4(a). For numerical estimations the following values of the laser heterostructure parameters were used $\beta=10^{-}$ ${ }^{3}, \quad \alpha_{i}=10^{-10} \mathrm{~cm}^{2}, \quad w=10 \mu \mathrm{m}, l=250 \mu \mathrm{m}$, $\rho=5 \mathrm{~cm}^{-1}, \eta_{1}=0.37, \eta_{2}=0.18, \eta_{3}=0.17$, $\eta_{4}=0.28$. Typical tuning curves, i. e., the output $P$ versus on the lasing wavelength $\lambda$ for different levels of excitation of the laser diode are shown in Fig. 4(b). The tuning range broadens with increasing the pump current. Note, that the operation wavelength does not obligatory correspond to the maximum in the modal gain spectrum. Continuous tuning at the output powers up to $4 \mathrm{~mW}$ is possible in the wavelength interval of 800 to $850 \mathrm{~nm}$.

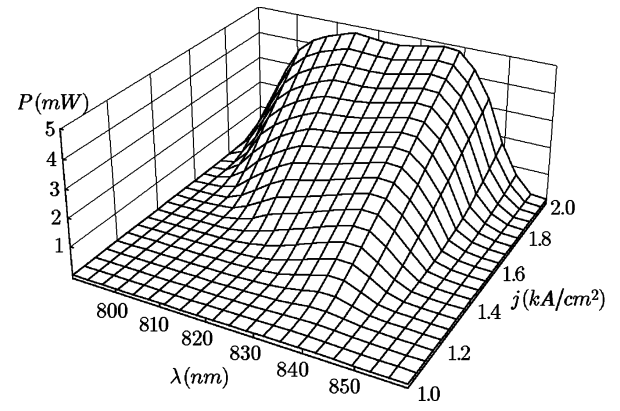

Fig. 5. Output power versus wavelength and current density for the tunable laser diode in the external cavity.

Fig. 4. (a) Light-current characteristic of the asymmetric multiple quantum-well heterostructure laser at different wavelengths, (- -) $\lambda=810,(-) 825,(-$. -$) 840 \mathrm{~nm}$. (b) Tuning curves of the laser at different pump current densities (1) $j=1.6$, (2) 1.7 , (3) 1.8 , and (4) $1.9 \mathrm{kA} / \mathrm{cm}^{2}$. 
Tuning characterization of the laser spectrometer in the $\lambda-j$-space is presented in Fig. 5. Selection by the angle of rotation of the tuning mirror provides optimal output power and required spectral interval in the region of 790 to $860 \mathrm{~nm}$. The use of other ternary or quaternary semiconductor compounds instead of GaAs in the active region gives possibilities to transfer the tuning curve to a necessary spectral range.

\section{Conclusion}

For novel type of tunable laser diodes based on asymmetric multiple quantum-well heterostructures with non-uniform excitation, it is shown that the continuous tuning of the lasing wavelength at constant output power is possible in a broad spectral range. The tuning range reaches up about $50 \mathrm{~nm}$ and increases with growing the pump current density. The described laser structures are suitable for the creative of effective tunable sources of radiation. Novel tunable laser sources can be used for a wide variety of applications such as coherent light spectroscopy, chemical analysis, metrology, and monitoring.

\section{Acknowledgements}

The work was supported by the Belarussian Republican Foundation for Fundamental Research.

\section{References}

[1] T. Day, F. Luecke, and M. Brownell, Lasers \& Optronics, 13 (1993) 15.

[2] W. Streifer, D.R. Scifres, G.L. Harnagel, D.F. Welch, J. Berger, and M. Sakamoto, IEEE J. Quantum Electron., 24 (1988) 883.
[3] T.Y. Fan, G. Huber, R.L. Byer, and P. Mitzscherlich, IEEE J. Quantum Electron., 24 (1988) 924.

[4] A.I. Nadezhdinskii and A.M. Prokhorov, Proc. SPIE, 1724 (1992) 2.

[5] D.A.V. Kliner, J.P.Koplow, and L. Goldberg, Opt. Lett., 22 (1997) 1418.

[6] D.W. Hughes and J.R.M. Barr, J. Phys. D:Appl. Phys., 25 (1992) 563.

[7] J.Z. Wilcox, S. Ou, J.J. Yang, M. Jansen, and G.L. Peterson, Appl. Phys. Lett., 54 (1989) 2174.

[8] I.S. Manak, A.A. Afonenko, V.K. Kononenko, S.V. Nalivko, and V.A. Shevtzov, Proc. SPIE, 3176 (1997) 345.

[9] V.K. Kononenko, Proc. SPIE, 1724 (1992) 89.

[10] V.K. Kononenko, I.S. Manak, S.V. Nalivko, V.A. Shevtsov, and D.S. Shulyaev, J. Appl. Spectrosc., 64 (1997) 234.

[11] J. Huang and L.W. Casperson, Opt. \& Quantum Electron., 25 (1993) 369.

[12] M. Asada, IEEE J. Quantum Electron., 25 (1989) 2019.

[13] S. Das Sarma, R. Jalabert, and S.-R. E. Yang, Phys. Rev. B, 41 (1990) 8288.

[14] M.G. Littman and H.J. Metcalf, Appl. Opt., 17 (1978) 2224.

[15] D. Wandt, M. Laschek, A. Tünnermann, and H. Welling, Opt. Lett., 22 (1997) 390.

[16] W. Kaenders, EuroPhotonics, 3 (1998) 30.

[17] V.M. Chulanovskii, Introduction to Molecular Spectral Analysis, Gostehisdat, Moscow-Leningrad, 1951.

[18] V.M. Kolesnikov, Optical Elements, Materials and Mediums of IR Technics, BSU, Minsk, 1983. 